\title{
Encapsulation of Photosystem I in Organic Microparticles Increases Its Photochemical Activity and Stability for Ex Vivo Photocatalysis
}

\author{
Arianna Cherubin, ${ }^{\dagger}$ Laura Destefanis, ${ }^{\dagger}$ Michele Bovi, $^{\dagger}$ Federico Perozeni, $^{\dagger}$ Ilaria Bargigia, ${ }^{\ddagger}, \S$ \\ Gabriel de la Cruz Valbuena, ${ }^{\ddagger} \|$ Cosimo D’Andrea, ${ }^{\ddagger}, \|$ Alessandro Romeo, ${ }^{\perp}$ Matteo Ballottari, ${ }^{*}, \uparrow$ \\ and Massimiliano Perduca* ${ }^{\dagger}$ \\ ${ }^{\dagger}$ Department of Biotechnology, University of Verona, Strada Le Grazie 15, 37134 Verona, Italy \\ ${ }^{\ddagger}$ Center for Nano Science and Technology @PoliMi, Istituto Italiano di Tecnologia, via Pascoli 70/3, 20133 Milano, Italy \\ ${ }^{\S}$ Georgia Institute of Technology, School of Chemistry and Biochemistry, 901 Atlantic Drive, Atlanta, Georgia 30332-0400, United \\ States \\ "Department of Physics, Politecnico di Milano, P.za L. da Vinci 32, 20133 Milano, Italy \\ ${ }^{\perp}$ Department of Computer Science, University of Verona, Strada Le Grazie 15, 37134 Verona, Italy
}

Supporting Information

ABSTRACT: Photosystem I (PSI) is a pigment binding multisubunit protein complex involved in the light phase of photosynthesis, catalyzing a light-dependent electron transfer reaction from plastocyanin to ferredoxin. PSI is characterized by a photochemical efficiency close to one, suggesting its possible application in light-dependent redox reaction in an extracellular context. The stability of PSI complexes isolated from plant cells is however limited if not embedded in a protective environment. Here we show an innovative solution for exploiting the photochemical properties of PSI, by encapsulation of isolated PSI complexes in PLGA (poly lactic-co-glycolic acid) organic microparticles. These encapsulated PSI complexes were able to catalyze light-dependent

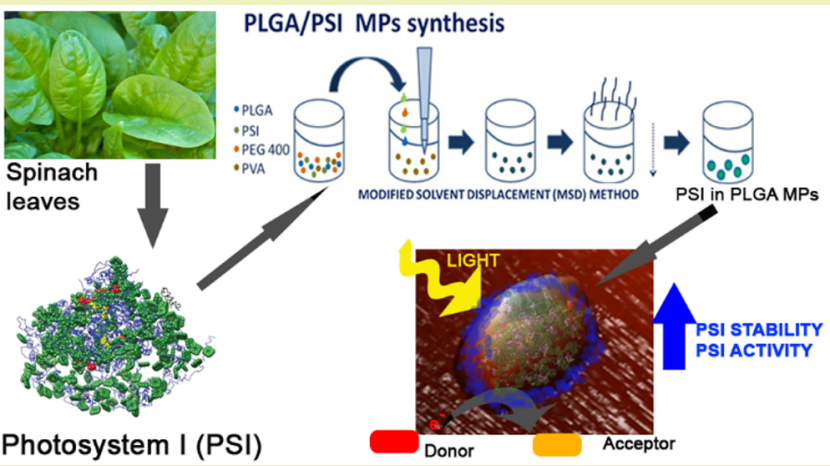
redox reactions with electron acceptors and donors outside the PLGA microparticles. Moreover, PSI complexes encapsulated in PLGA microparticles were characterized by a higher photochemical activity and stability compared with PSI complexes in detergent solution, suggesting their possible application for ex vivo photocatalysis.

KEYWORDS: Photochemistry, Photocatalysis, Photosynthesis, Microparticles, Chitosan, Poly lactic-co-glycolic acid

\section{INTRODUCTION}

Photosystems (PS) are membrane protein complexes that bind pigments and are responsible for light harvesting and its conversion into chemical energy by promoting a lightdependent electron transport chain across the photosynthetic membranes. ${ }^{1-4}$ In eukaryotic photosynthetic organisms, two photosystems work in series, photosystem I (PSI) $)^{1,5,6}$ and photosystem II (PSII), with PSI being a plastocyaninferredoxin light-dependent oxidoreductase and PSII a lightdependent water-plastoquinone oxidoreductase. ${ }^{3}$ PSI and PSII are then connected by the protein complex called Cytochrome $b_{6} \mathrm{f}$ which oxidizes plastoquinones and reduces plastocyanin. ${ }^{7}$ Both PSI and PSII are composed of a core complex hosting the reaction center where charge separation occurs and an outer antenna system made of light-harvesting complexes. ${ }^{8,9}$ In eukaryotic organisms, PSI is present in the thylakoid membrane as a monomer carrying its antenna moiety on one side in a "half-moon" shaped structure. ${ }^{1}$ The reaction-center core complex is made up of the heterodimeric PsaA and PsaB subunits, containing the primary electron donor, a chlorophyll dimer called P700, which undergoes light-induced charge separation and transfers an electron through the sequential carriers A0, A1, FX being respectively a monomeric chlorophyll a, two phylloquinones, and a $[4 \mathrm{Fe}-4 \mathrm{~S}]$ ironsulfur center. ${ }^{1}$ The final acceptors, FA and FB, two other $[4 \mathrm{Fe}-4 \mathrm{~S}]$ iron-sulfur center, are located on another subunit, PsaC. ${ }^{1}$ The redox potential of the primary donor, P700, is $+0.43 \mathrm{~V}$ and that of the final acceptor, $\mathrm{FB}$, is $-0.53 \mathrm{~V}$, producing a redox difference of $-1.0 \mathrm{~V}$. PSI absorbs in the $350-500 \mathrm{~nm}$ and $620-710 \mathrm{~nm}$ wavelength range: photochemical properties of PSI have been extensively studied either in intact cells or in detergent solution, revealing its extremely high quantum efficiency, close to 1 , with a very short trapping time of excitation energy at the reaction center $(30-70$

Received: February 4, 2019

Revised: April 19, 2019

Published: May 16, 2019 
ps), ${ }^{8,10,11}$ and an energy yield of $\sim 58 \%{ }^{12}$ The extremely high photochemical properties of PSI suggested its possible application in extracellular artificial devices as light convertor for the generation of electron transport chain ${ }^{12-22}$ or for ex vivo photocatalysis of redox reactions. ${ }^{17,23}$ Moreover, PSI photochemical properties could also be potentially used for photoactivation of mammalian cells: ${ }^{24}$ optical stimulation of living cells and tissues by means of micro- and nanoparticles is an extremely promising tool in medicine and biology in order to control neuronal networks or physiological functions. ${ }^{25}$ The different applications of PSI require its assembly in stable systems. Previous reports demonstrated the importance of the environment for the photochemical properties of PSI, ${ }^{26,27}$ which was reported to be successfully improved upon encapsulation in various organic/inorganic interfaces. ${ }^{14,28-37}$ In order to design an environmentally friendly photocatalytic system which could be also used for applications in mammalian cells, a biocompatible formulation of PSI is required. Micro- or nanoparticles can be applied, thanks to their small size and the protected environment they offer, to fibers, sensors, wires, sheaf, meshes, cosmetic, medical, diagnostic, and therapeutic fields. ${ }^{38}$ Among the organic polymers for particles synthesis, chitosan and PLGA are interesting for their safety and biocompatibility. ${ }^{38-44}$ Chitosan is a cationic amino polysaccharide copolymer of $\beta$-(1-4) linked D-glucosamine and $\mathrm{N}$-acetyl-glucosamine derived from chitin by alkaline or enzymatic partial deacetylation. ${ }^{44}$ PLGA is a biocompatible copolymer of poly lactic acid (PLA) and poly glycol acid (PGA) that is used to synthesize biodegradable micro or nanoparticles. ${ }^{38,40,41,43,45}$ Thanks to the rapid degradation without negative side effects on the human body and the absence of significant toxicity, the U.S. Food and Drug Administration (FDA) and the European Medicine Agency (EMA) have approved both chitosan and PLGA particles as delivery vehicle for drugs, proteins, and other molecules such as DNA, RNA, and peptides. ${ }^{38,40,41}$ These kinds of micro and nanoparticles offer a nonpolar environment protecting hydrophobic compounds from water contact and mimicking what happens in cellular membranes and organelles. In this work, we investigated the possibility to encapsulate PSI complexes isolated from spinach leaves in organic microparticles (MPs) in order to obtain a stable and efficient light-dependent redox catalyst.

\section{MATERIALS AND METHODS}

Photosystem I Purification. Photosystem I complexes were purified from spinach leaves as described in ref 46. In particular, thylakoid membranes were purified from spinach leaves and then solubilized in $0.8 \%$ dodecyl $\beta$-D-maltoside, and the solubilized membranes were loaded on sucrose gradient in order to separate the different pigment binding complexes by ultracentrifugation. Isolated PSI were kept in $0.5 \mathrm{M}$ sucrose, Hepes $20 \mathrm{mM} \mathrm{pH} 7.5$, and $0.03 \%$ dodecyl $\beta$-D-maltoside: this buffer was also adopted for all the measurement of PSI in detergent conditions.

Synthesis of Poly Lactic-co-glycolic Acid Microparticles. The modified solvent displacement method (MSD) was used in PLGA microparticles preparation ${ }^{45}$ (Supporting Information, Figure S1): a polymeric phase containing $500 \mu \mathrm{L}$ of PEG 400 (Merck-Schuchardt), $500 \mu \mathrm{L}$ of poly(vinyl alcohol) (PVA) $1 \%$ solution, $2 \mathrm{~mL}$ of PSI (chlorophylls concentration $89.1 \mu \mathrm{g} / \mathrm{mL}$ in the sample), and $20 \mathrm{mg}$ of PLGA 75:25 (Sigma) was sonicated for $1 \mathrm{~min}$ (5 $\mathrm{s}$ of sonication alternated to $5 \mathrm{~s}$ of time pausing) and slowly added dropwise to an aqueous phase of $12 \mathrm{~mL}$ of $1 \%$ PVA (Sigma). Sonication power used was $8 \mathrm{~W}$. The mixture was sonicated $4 \mathrm{~min}$ at the same rate. The whole preparation was kept under magnetic stirring for $6 \mathrm{~h}$, and the solution obtained was centrifuged for $20 \mathrm{~min}$ at $12000 \mathrm{~g}$; the pellet was resuspended in $15 \mathrm{~mL}$ of Milli-Q water, and the solution was dialyzed against deionized water overnight, with a cellulose membrane, cutoff $10-14 \mathrm{kDa}$ (Sigma), to eliminate the excess PEG. ${ }^{39}$ Two washing cycles with Milli-Q water were done to eliminate the excess PEG and the PSI, which has not been encapsulated; hence, microparticle solution was washed by centrifugation for $15-20 \mathrm{~min}$ at $12000 \mathrm{~g}$, until the supernatant was transparent because of the absence of PSI (as confirmed by absorption measurements at $680 \mathrm{~nm}$ ). Empty microparticles were prepared following the same protocol, but the volume of PSI solution was replaced by PVA 1\%. All samples were lyophilized and resuspended in Milli- $Q$ water at the proper concentration required for each experiment.

Synthesis of Chitosan Microparticles. Chitosan microparticles were prepared via the ionic cross-linking method, ${ }^{44}$ in which an anionic cross-linker, such as tripolyphosphate (TPP, Sigma), is used (Supporting Information, Figure S5). In particular, $3 \mathrm{~mL}$ of TPP solution at $0.028 \%$ was added dropwise in $5 \mathrm{~mL}$ of chitosan solution $0.1 \%$, in which $100 \mu \mathrm{g}$ (chlorophylls concentration of $89.1 \mu \mathrm{g} / \mathrm{mL}$ ) of PSI were added, under magnetic-stirring. Magnetic-stirring was then kept constant for half an hour, and a solution of microparticles was then washed twice by centrifugation for $15 \mathrm{~min}$ at $12000 \mathrm{~g}$, while the pellet was suspended in $500 \mu \mathrm{L}$ of Milli- $\mathrm{Q}$ water. All samples were lyophilized and resuspended in Milli- $Q$ water at the proper concentration required for each experiment.

Dynamic Light Scattering Analysis. The PLGA MPs size and the Z-potential were estimated using the DLS technique (Nano ZetaSizer ZS, ZEN3600, Malvern Instruments, Malvern, Worcestershire, U.K.). All the analyses were performed at $25{ }^{\circ} \mathrm{C}$ by resuspending the synthesized microparticles in distilled water.

Differential Scanning Calorimetry (DSC) Analysis. Differential scanning calorimetry (DSC) experiments were performed on a NanoDSC, TA Instruments (New Castle, Delaware, U.S.A.). Isothermal mode was used to evaluate the heat release due to the breakdown of the PLGA shell or due to the dissolution of the protein structure of the PSI. The experiments were run at a fixed temperature of $4{ }^{\circ} \mathrm{C}$ maintained overnight for the PSI samples and over the weekend for the encapsulated PSI. Experimental setup and data collections were managed with the DSCRun software (TA Instruments).

Atomic Force Microscopy (AFM). The synthesized microparticles were analyzed and characterized using the atomic force microscope (NT-MDT Solver Pro microscope, NT-MDT Spectrum Instruments, Zelenograd, Moscow, RU), with a single-crystal siliconantimony-doped probe and a gold-coated base (NSG-01 from NTMDT). To reduce nonlinearity and hysteresis in the measurements, prior to the AFM analyses the microscope was calibrated by a calibration grid (TGQ1 from NT-MDT). The analyses were performed using the tapping mode where the cantilever is driven to oscillate vertically near its resonance frequency. The lyophilized sample was resuspended in Milli- $Q$ water, deposited on a mica substrate, and dried under a flux of argon. All the data were collected using the software Nova (NT-MDT). The resolution of the images acquired was $15 \mathrm{~nm}$.

P700 Activity. PSI photochemical activity was measured following the kinetics of P700 oxidation. In particular, P700 oxidation upon actinic light illumination was analyzed measuring pump-probe transient absorption at $705 \mathrm{~nm}$ as previously described, using a JTS 10-LED pump-probe spectrometer (Bio-Logic SAS, Claix, France). ${ }^{47}$ P700 oxidation kinetics were measured on PSI complexes in detergent

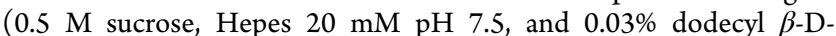
maltoside) or in MPs (resuspended in water) at a chlorophyll concentration of $20 \mu \mathrm{g} / \mathrm{mL}$ applying an actinic orange light at 940 $\mu \mathrm{mol} \cdot \mathrm{m}^{-2} \cdot \mathrm{s}^{-1}$. In the specific cases described in the text, electron donor and acceptor molecules (ascorbate and methyl-viologen respectively) were added to the MPs at a concentration of $1 \mathrm{mM}$. Ascorbate was used as electron donor because of its slower rate of $\mathrm{P} 700^{+}$reduction compared to electron transfer to methyl-viologen or 

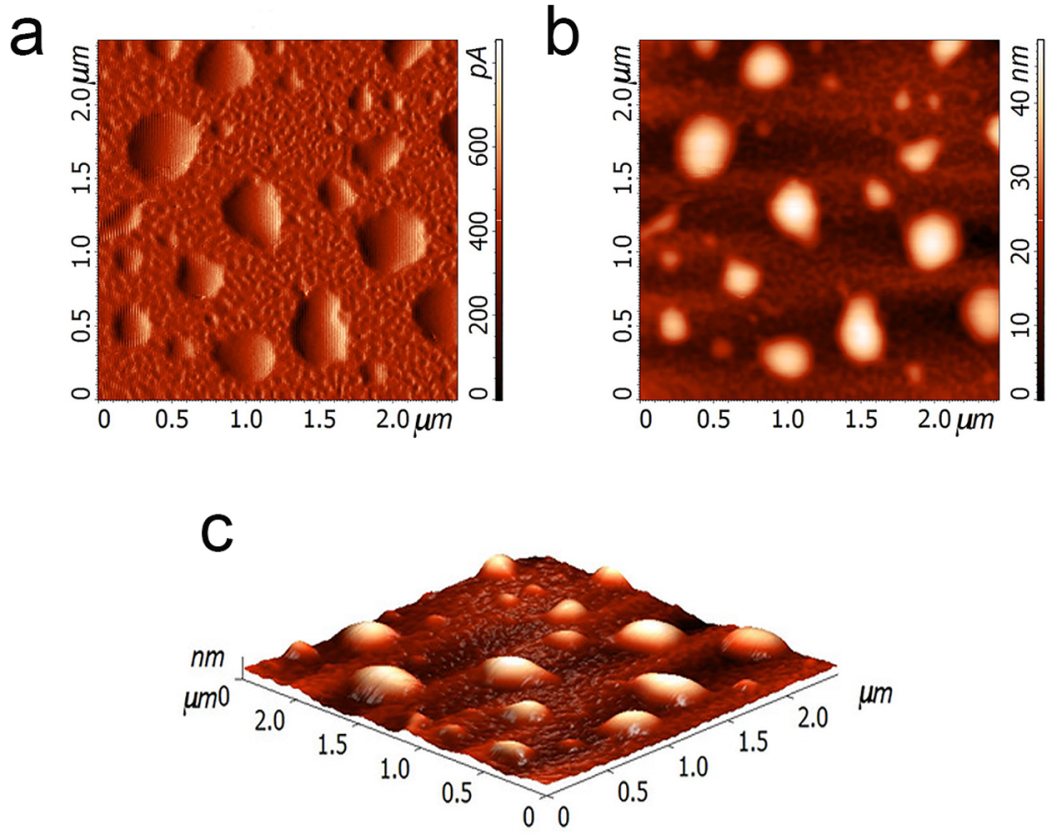

Figure 1. Atomic force microscopy (AFM) observation of PSI in PLGA microparticles. PLGA MPs visualized in three different modes: (a) Errormode; (b) Semicontact; (c) 3D. The images have been acquired in dry mode.

charge recombination: in this way, the extent of ${\mathrm{P} 700^{+}}^{+}$was determined by the efficiency of PSI to donate electron to acceptors.

Fluorescence Lifetime Measurements. Time-resolved fluorescence measurements were performed using a femtosecond laser excitation at $440 \mathrm{~nm}$ and a streak camera detection system, as reported in ref 47 . Briefly, laser pulsed at $440 \mathrm{~nm}$ operating at 80 $\mathrm{MHz}$ with temporal and spectral bandwidths of $140 \mathrm{fs}$ and $5 \mathrm{~nm}$, respectively, were focused onto the sample, maintaining a low laser power intensity $(50 \mu \mathrm{W}, 100 \mu \mathrm{m}$ spot diameter). The samples were kept at a constant temperature of $11{ }^{\circ} \mathrm{C}$ by a temperature-controlled cuvette cooled by a Peltier system. The resulting collected emission was analyzed by a spectrograph (Princeton Instruments Acton SP2300) coupled to a streak camera (Hamamatsu C5680) equipped with a synchroscan voltage sweep module with spectral and temporal resolutions of $\sim 1 \mathrm{~nm}$ and $\sim 3 \mathrm{ps}$, respectively. Temporal broadening of the pump pulses caused by dispersive elements was confirmed to be well below the response time of the detection system. Fluorescence decay maps were then integrated in the $0-1800$ ps time range in order to obtain the integrated spectra and in the 600-800 nm wavelength range for determining the integrated decay kinetics. Integrated decay kinetics were fitted with two (PSI in MPs) or three (PSI in detergent) exponential forms, whose amplitudes $\left(\mathrm{A}_{x}\right)$ and decay constants $\left(\tau_{x}\right)$ were used to calculate the average fluorescence decay, $\tau_{\mathrm{AV}}$ as $\tau_{\mathrm{AV}=} \Sigma \delta_{x} * \tau_{x} / \Sigma \mathrm{A}_{x}$. In the case of PSI in detergent, the long decaying component with time constant in the ns time range was not considered for $\tau_{\mathrm{AV}}$ calculation, as previously reported, being related to free chlorophylls or antenna proteins in the PSI sample. Photochemical efficiency of PSI was calculated from $\tau_{\mathrm{AV}}$ as reported in ref 48 .

PSI Stability. PSI stability was measured in terms of maximum P700 oxidation level during high light $\left(1500 \mu \mathrm{mol} \mathrm{m}^{-2} \mathrm{~s}^{-1}\right)$ exposure for several days. PSI in detergent or in MPs were kept at room temperature in agitation exposed to LED white light $\left(1500 \mu \mathrm{mol} \mathrm{m}^{-2}\right.$ $\mathrm{s}^{-1}$ ) for up to 34 days. No volume loss was observed during treatment. P700 photochemical activity was measured as described above in the presence of ascorbate and methyl-viologen $(1 \mathrm{mM})$ added fresh for each measurement.

Pigments Analysis. The composition of the pigments present in PSI samples was analyzed after extraction with acetone $80 \%$; microparticles were lyophilized and then resuspended in $400 \mu \mathrm{L}$ of DMSO (DMSO temperature was about $65{ }^{\circ} \mathrm{C}$ ) to extract the pigments of interest. The extracts were analyzed by RP-HPLC as described in ref 49.

Statistical Analysis. All the synthesis and measurements reported in this work were performed in three independent replicates with three technical replicates each. Error bars are reported as standard deviation.

Availability of Data and Materials. All the data and materials generated and/or analyzed during the current study are available from the corresponding author on reasonable request.

\section{RESULTS}

Photosystem I Encapsulation in PLGA Microparticles. PSI supercomplexes were purified from spinach leaves as described in ref 46. Isolated PSI complexes were then encapsulated in PLGA microparticles (MPs) as described in the Methods section (Supporting Information, Figure S1). PLGA polymer was initially chosen for MPs production because of the negative charges present in this molecule, which could potentially interact with oxidized $\mathrm{P} 700^{+}$. The resulting MPs, after several washing steps, were green, and the pigment composition of this MPs was investigated by HPLC. As reported in Supporting Information, Table S1, the pigment content of PSI in PLGA MPs was like the pigments identified in PSI in detergent solution, suggesting the encapsulation procedure did not significantly alter the pigment binding properties of the PSI complex. Encapsulation efficiency, calculated on the basis of the analysis on the content of chlorophylls in microparticles, was $47.2 \%$, indicating that the microparticles offer a suitable environment to the PSI encapsulation. In addition, from the chlorophyll content per gram of PLGA MPs, it was possible to calculate a PSI content per gram of PLGA MPs of $99 \mu \mathrm{mol} \mathrm{PSI} / \mathrm{g}$ particles. Size and superficial charge of PLGA MPs containing PSI were then investigated by dynamic light scattering (DLS) compared to PSI in detergent. PSI in detergent solution was quite homogeneous with an average size of about $55 \mathrm{~nm}$ and a PDI (polydispersity index) of 0.06 (Supporting Information, Table S2). It is important to note that DLS measurements of 

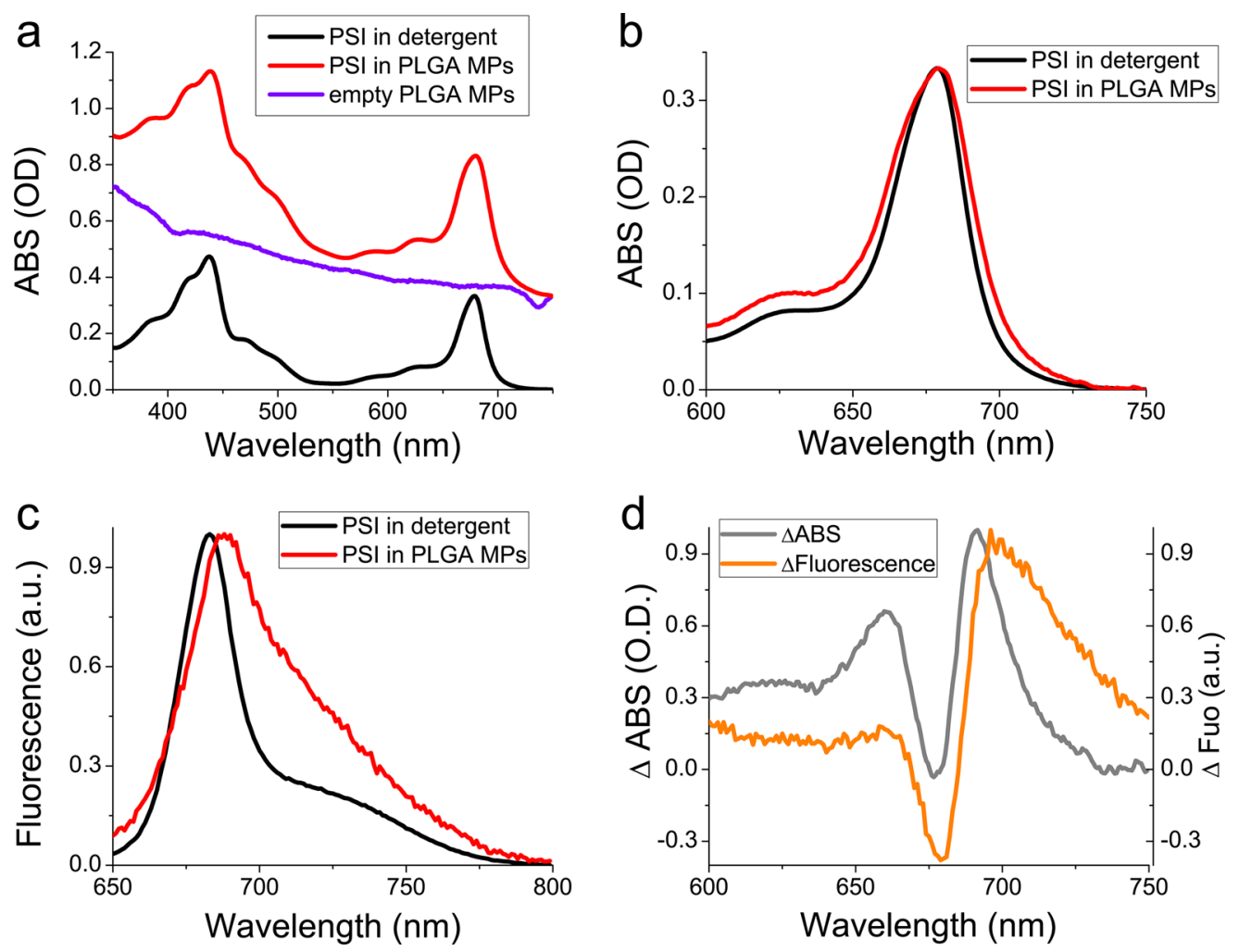

Figure 2. Absorption and fluorescence emission spectra of PSI in detergent and in PLGA microparticles. (a) Absorption spectra of isolated PSI in detergent solution (black) or in MPs (red). The scattering traces of empty PLGA MPs is also reported in blue. (b) Absorption spectra of PSI in detergent solution vs PSI in PLGA MPs in the $600-750 \mathrm{~nm}$ range. Absorption spectrum of PSI in PLGA MPs was obtained after subtraction of the scattering trace of empty MPs. Absorption spectra were normalized to the maximum absorption in the 600-750 nm region. (c) Fluorescence emission spectra of PSI in detergent vs PSI in PLGA MPs upon excitation at $440 \mathrm{~nm}$ (similar results were obtained upon excitation at 475 or 500 $\mathrm{nm}$ ). Fluorescence emission spectra were normalized to the maximum peak in the $600-750 \mathrm{~nm}$ region. (d) Difference absorption (gray) or fluorescence (orange) spectra obtained as PSI in PLGA MPs minus PSI in detergent.

PSI in detergent give information about the detergent micelles formed around PSI complexes, increasing the apparent diameter of the complexes from $\sim 20 \mathrm{~nm}$ (isolated PSI) ${ }^{1}$ to $55 \mathrm{~nm}$ (PSI in detergent micelles). The empty microparticles suspension was homogeneous with an average size of $402 \mathrm{~nm}$ and a PDI of 0.02, thus confirming the uniformity of the suspension. Not surprisingly, the suspension of PSI containing PLGA MPs was inhomogeneous, with an average size of 1700 $\mathrm{nm}$ and a PDI of 0.5: encapsulated PSI might establish different interactions with the PLGA matrix and different MPs might contain different numbers of PSI complexes, giving rise to particles having inhomogeneous size. Moreover, microparticles can aggregate together, and this can affect DLS analysis. The superficial charge measured for PLGA MPs was negative, strictly connected to the chemical characteristics of the polymeric PLGA (Supporting Information, Table S2). Indeed, the amphiphilic nature of the PLGA monomers forces the polar carboxylic groups of PLGA to be exposed at the water solvent during the microparticles synthesis. The encapsulation of PSI in PLGA MPs was further confirmed by AFM measurements, showing that the empty PLGA MPs were much smaller compared with PLGA MPs containing PSI (Supporting Information, Figure S2). Moreover, PSI containing PLGA MPs looked like spheres with well-defined outlines and smooth surface (Figure 1), suggesting that all the PSI was encapsulated and not attached outside the shell. This finding is also consistent with the hydrophobicity of PSI complexes, leading to a reduced exposure to the water solvent.
Photosystem I Encapsulation in PLGA Microparticles: Absorption and Fluorescence Emission Spectra. To verify the presence of PSI in PLGA MPs and their optical properties, absorption and fluorescence emission spectra were acquired. PLGA MPs assembled in the presence of PSI were clearly characterized by absorption peaks in the $350-500 \mathrm{~nm}$ and $615-720 \mathrm{~nm}$, as expected in the presence of chlorophyll binding complexes (Figure 2). When the absorption spectrum of PSI in PLGA MPs was corrected for the scattering due to the dimension of the MPs, subtracting the signal obtained for empty PLGA MPs, it was possible to compare the absorption of encapsulated PSI vs PSI in detergent solution: PSI in PLGA MPs presented similar absorption characteristics of the PSI in detergent but exhibiting a broader peak in the $600-750 \mathrm{~nm}$ region, indicating the formation of stronger Chl-Chl interaction in PLGA MPs, which is likely due to a protein aggregation, with a consequent enlargement of the absorption spectrum. ${ }^{50}$ In agreement with this observation, fluorescence emission spectra of PSI were characterized by a red-shift of the peak from $684 \mathrm{~nm}$ (in detergent) to $689 \mathrm{~nm}$ (in PLGA MPs) and by a stronger emission in the $700-750 \mathrm{~nm}$ region for PSI in PLGA MPs compared with PSI in detergent. The fluorescence emission above $700 \mathrm{~nm}$ in PSI complexes is due to energy transfer to low energy chlorophylls commonly named "red forms" which are strongly coupled forming a charge transfer state: ${ }^{51}$ the red-shifted absorption and increased emission above $700 \mathrm{~nm}$ in PLGA MPs (Figure 2) 

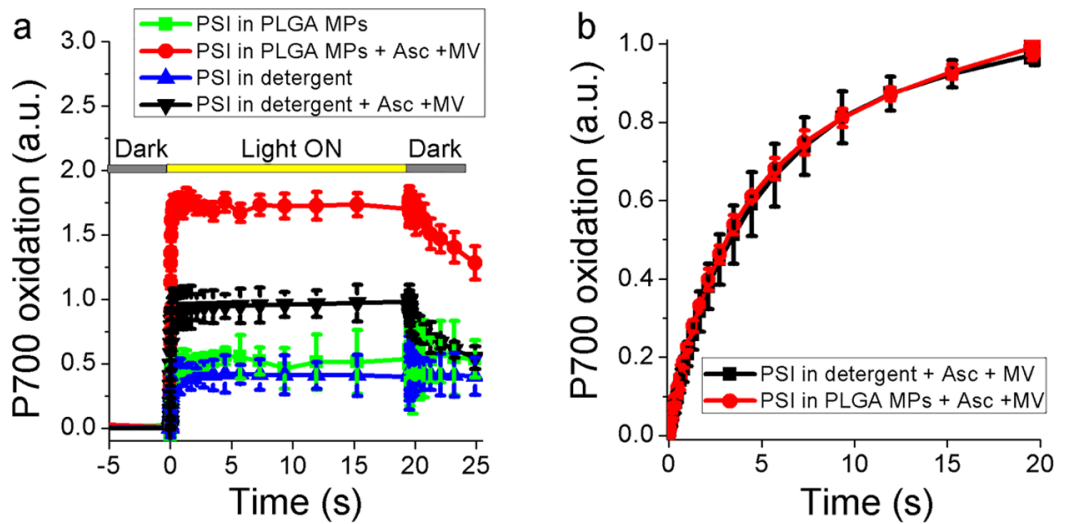

Figure 3. Photochemical activity of PSI. (a) Light-dependent P700 oxidation of PSI in detergent or in PLGA MPs measured as transient absorption at $705 \mathrm{~nm}$. Ascorbate (Asc) and methyl-viologen (MV) were added where indicated as electron donor and acceptor, respectively. Orange actinic light at $940 \mu \mathrm{mol} \mathrm{m}^{-2} \mathrm{~s}^{-1}$ were used to induce P700 oxidation. P700 oxidation kinetics were normalized to the chlorophyll content of each sample. (b) Light-dependent P700 oxidation measured under limiting light $\left(12 \mu \mathrm{mol} \mathrm{m}^{-2} \mathrm{~s}^{-1}\right)$ in the presence of electron donors and acceptors. Kinetics traces were in this case normalized to the maximum value. In these conditions, the kinetics of P700 oxidation are inversely proportional to PSI light-harvesting properties.
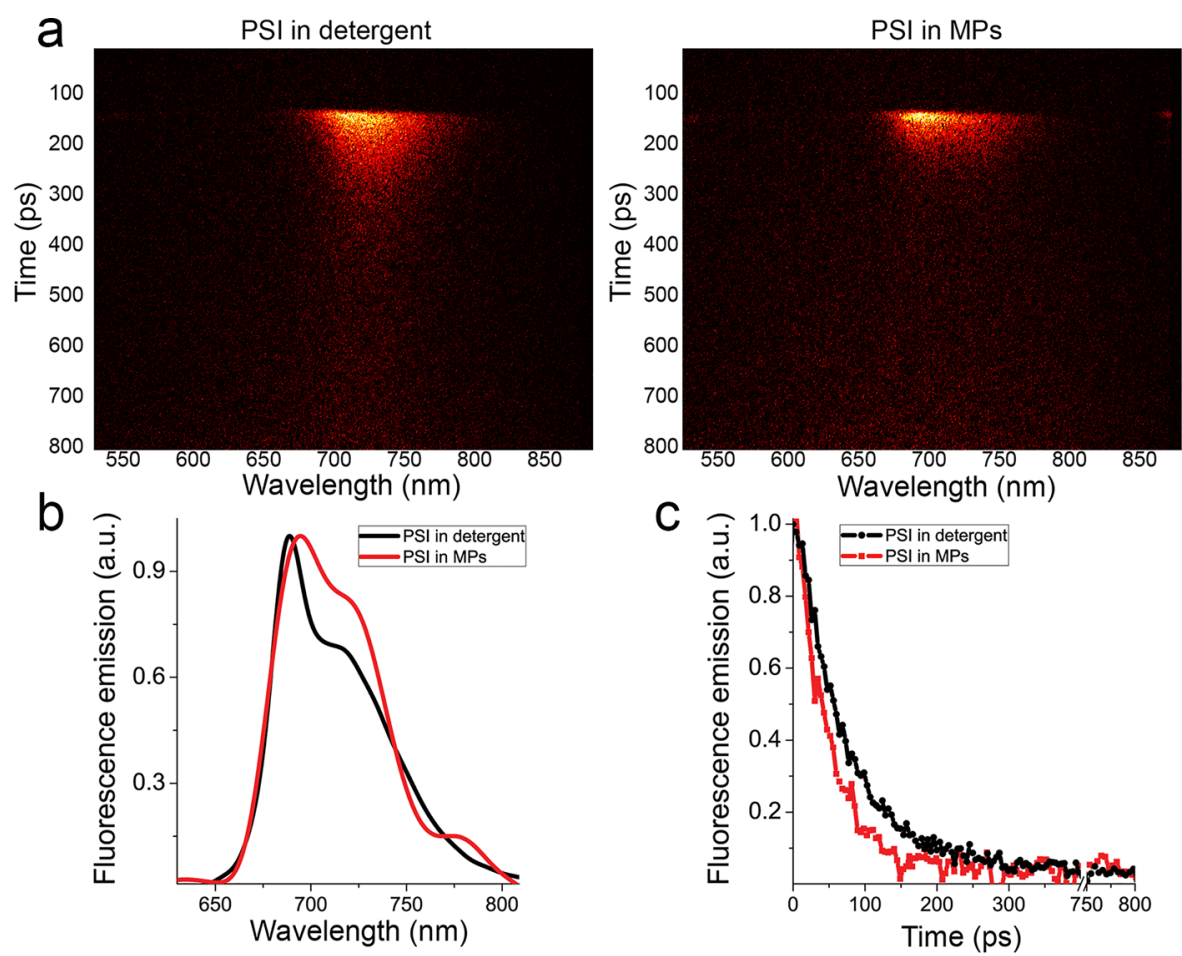

Figure 4. Time-resolved analysis of PSI in detergent or in PLGA microparticles. (a) Fluorescence decay maps obtained upon laser excitation (440 $\mathrm{nm}$ ) of PSI in detergent or in MPs. (b) Integrated spectra in the 0-1800 ps time range. (c) Integrated kinetics in the 650-800 nm wavelength range.

might thus be attributed to a stronger Chl-Chl interaction likely caused by protein aggregation in MPs.

Photosystem I Photochemistry in PLGA Microparticles. PSI photochemical activity was measured in detergent and in PLGA MPs to evaluate the possible charge separation at the level of its reaction center, P700. Photochemical activity of PSI can be measured following the kinetics of P700 oxidation upon light exposure. Reduced P700 indeed has a maximum absorption peak at $\sim 705 \mathrm{~nm}$, while the oxidized form $\mathrm{P} 700^{+}$ has red-shifted absorption with a peak at $\sim 830 \mathrm{~nm}$ : kinetics of P700 oxidation can thus be obtained following the transient absorption decrease at $705 \mathrm{~nm}$ upon exposure of PSI to an actinic light. ${ }^{52}$ As reported in Figure 3a, both PSI in detergent solution and in PLGA MPs exhibited a significant P700 oxidation in actinic light, which was further increased in the presence of ascorbate and methyl viologen (MV) in solution as electron donor and electron acceptor, respectively. When the actinic light was switched off, $\mathrm{P} 700^{+}$was rereduced only in the presence of ascorbate. Interestingly, upon normalization of P700 oxidation kinetics to the total chlorophyll content, PSI in PLGA MPs was characterized by a $\mathrm{P} 700^{+}$formation increase of $\sim 75 \%$ compared with the same complex in detergent solution. To evaluate possible different light-harvesting properties of PSI in PLGA MPs compared to detergent, the functional antenna size of PSI was evaluated by measuring the kinetics of P700 oxidation in limiting light, ${ }^{53}$ where the light-harvesting 

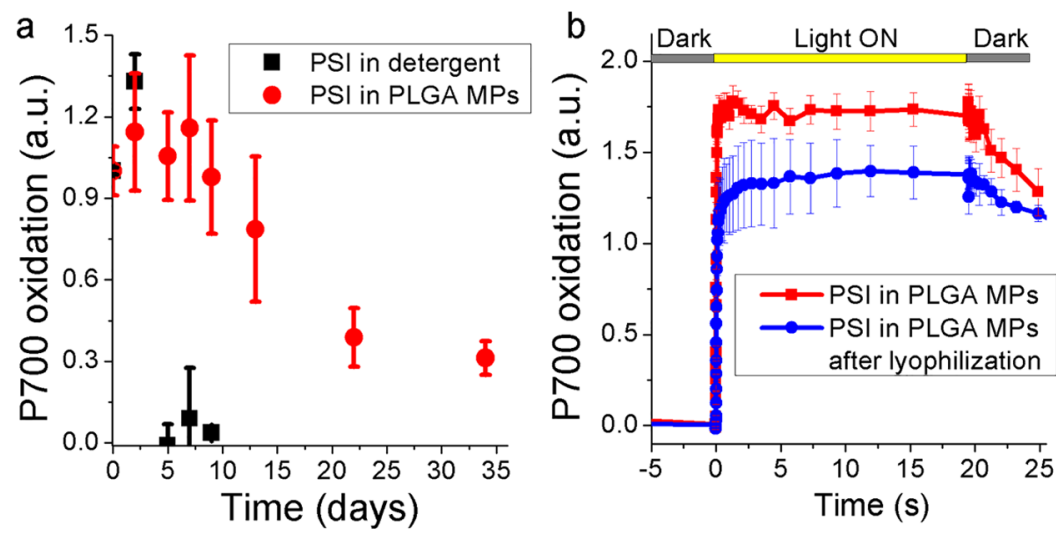

Figure 5. Stability of PSI in PLGA MPs to light exposure and lyophilization. (a) Maximum P700 activity measured at different time (days) of exposure to $1500 \mu \mathrm{mol} \mathrm{m} \mathrm{m}^{-2} \mathrm{~s}^{-1}$. (b) P700 oxidation measured in PSI in PLGA MPs before and after lyophilization, with the latter being resuspended in the same initial volume. Ascorbate and methyl-viologen were added fresh $(1 \mathrm{mM})$ during each measurement.

efficiency and the rate of P700 oxidation are inversely related. As reported in Figure $3 \mathrm{~b}$, the kinetics of P700 oxidation in limiting light, normalized to the maximum $\mathrm{P} 700^{+}$value, were similar for PSI in detergent or in PLGA MPs, indicating similar light-harvesting properties.

The photochemical efficiency of PSI in PLGA MPs was then investigated by measuring fluorescence lifetimes: an increased photochemical efficiency will decrease the fluorescence lifetime and fluorescence quantum yield because of increased nonradiative de-excitation. Time-resolved fluorescence for PSI in detergent solution or in PLGA MPs were acquired by a timeresolved fluorescence spectroscopy system based on a Streak Camera detector by which the fluorescence emission kinetics are acquired at different wavelengths of emission, with the resulting $2 \mathrm{D}$ fluorescence maps reported in Figure $4 \mathrm{a}$. The fluorescence emission spectra integrated in the time range $0-$ 800 ps confirmed the red-shifted emission for PSI in MPs compared to PSI in detergent (Figure 4b). As reported in Figure $4 c$, the integrated emission kinetics in the $650-800 \mathrm{~nm}$ emission range were faster in the case of PSI in PLGA MPs. Measurements of time-resolved fluorescence at different power intensity showed that the faster decay of fluorescence kinetics of PSI in PLGA MPs was not due to annihilation effect as reported in Supporting Information, Figure S3. Average fluorescence lifetimes $\left(\tau_{\mathrm{AV}}\right)$ calculated from exponential fitting of the decay traces resulted as 48.7 ps in the case of PSI in detergent and 31.5 ps for encapsulated PSI (Supporting Information, Table S3). These values can be approximated to the trapping time, the time required for the excitation energy to reach the reaction center, and can be used to calculate the photochemical efficiency of PSI as previously described, ${ }^{48}$ resulting in $98.5 \%$ and $99.1 \%$ photochemical efficiency for PSI in detergent and in PLGA MPs, respectively. ${ }^{48}$

Stability of Photosystem I in PLGA Microparticles. The potential advantage of having a PSI complex encapsulated in MPs is an increased stability due to a shell effect of the organic matrix. The stability of PSI in PLGA MPs was thus investigated by DSC and compared to PSI in detergent solution. As reported in Supporting Information, Figure S4, a negative signal was measured for either PSI in detergent or in PLGA MPs, indicating that the disruption of PSI complexes is an exothermic event. Clearly, this negative peak was delayed in time when PSI was encapsulated in PLGA MPs compared with PSI in detergent solution: this result indicates that the presence of the PLGA shell conferred a longer stability to PSI.
The increased stability of PSI in PLGA MPs was then evaluated measuring the PSI photochemical activity after different times of illumination with strong white light (1500 $\left.\mu \mathrm{mol} \mathrm{m} \mathrm{m}^{-2} \mathrm{~s}^{-1}\right)$. As reported in Figure 5, PSI in detergent solution completely lost its photochemical activity after 5 days of illumination, while PSI in PLGA MPs exhibited an almost unaltered photochemical activity for at least 10 days. A residual $30 \%$ photochemical activity was still measured in PSI in PLGA MPs after 34 days of illumination.

Encapsulation of PSI in Chitosan Microparticles. To evaluate if the increased photochemical activity and increased stabilization effect were specifically related to the chemical properties of the PLGA matrix, PSI encapsulation was also performed in chitosan MPs (Supporting Information, Figure S5). As reported in Supporting Information, Table S2, chitosan MPs were half the size compared with PLGA MPs but with a positive superficial charge. AFM observation of PSI in chitosan MPs yielded similar results compared to PLGA MPs (Supporting Information, Figure S6). Differently, the redshifted absorption spectrum and fluorescence emission above $700 \mathrm{~nm}$ were less pronounced compared with PLGA encapsulation, even if still evident (Supporting Information, Figure S7). Photochemical activity of PSI measured as $\mathrm{P}^{+} 00^{+}$ oxidation was increased compared with PSI in detergent but lower compared with PSI in PLGA MPs (Supporting Information, Figure S8). Stability of PSI in chitosan MPs was then evaluated as described above after different times of illumination with strong white light $\left(1500 \mu \mathrm{mol} \mathrm{m} \mathrm{m}^{-2} \mathrm{~s}^{-1}\right)$ : differently from the case of PSI in PLGA MPs, encapsulation in chitosan MPs did not increase its stability during light exposure (Supporting Information, Figure S9). The result obtained demonstrate that encapsulation in PLGA MPs is more efficient for preserving PSI photochemical activity and stability.

Photosystem I in PLGA Microparticles Is Resistant to Lyophilization. An important parameter to be considered for possible applications of PSI MPs is the stability to lyophilization since in many cases MPs need to be dried to extend their shelf life and/or for convenient storage/transportation. PLGA MPs containing PSI complexes were thus lyophilized, and the residual PSI photochemical activity was measured on both dried and resuspended PLGA MPs. As reported in Supporting Information, Figure S10, P700 oxidation was still detectable even on lyophilized PLGA MPs. Moreover, when lyophilized PLGA MPs were resuspended in water, the same absorption spectra (Supporting 
Information, Figure S11) were obtained comparing PSI in PLGA MPs before and after lyophilization. The photochemical activity of encapsulated PSI resuspended after lyophilization was then measured, upon addition of ascorbate and methylviologen as electron donor and acceptor, observing only a slight decrease compared to the PSI in PLGA MPs before lyophilization (Figure $5 \mathrm{~b}$ ): this result indicates that when PSI is encapsulated in the PLGA matrix, it can be lyophilized preserving most of its photochemical activity.

Photosystem I in PLGA Microparticles Can Catalyze Redox Reaction. Light-dependent P700 oxidation observed in PSI suggests its possible application as light-dependent catalyst for redox reaction. To prove this concept, encapsulated PSI in PLGA matrix was tested for possible reduction of methylene blue (Figure 6). Methylene blue is indeed

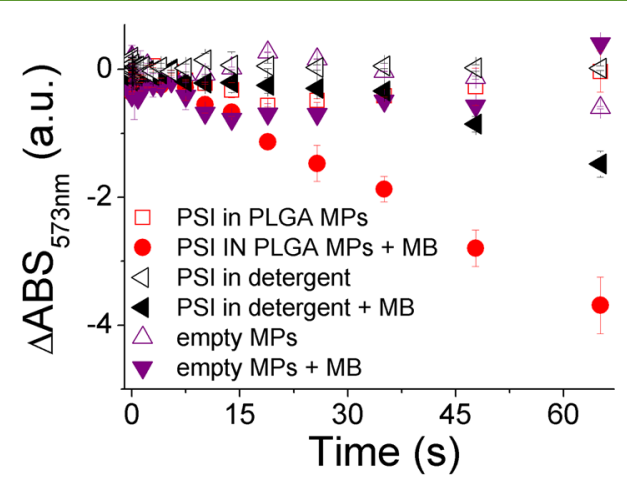

Figure 6. Light-dependent reduction of methylene blue mediated by PSI microparticles. Samples were illuminated at $940 \mu \mathrm{mol} \mathrm{m}^{-2} \mathrm{~s}^{-1}$. Transient absorption data are reported as the difference in absorbance at $537 \mathrm{~nm}\left(\triangle \mathrm{ABS}_{537 \mathrm{~nm}}\right)$, setting as 0 the $\Delta \mathrm{ABS}_{537 \mathrm{~nm}}$ value at time 0 .

commonly used as redox indicator, since its absorption in the 500-700 nm range decreases in the reduced form. ${ }^{54}$ When PSI in PLGA MPs were incubated with ascorbate as electron donor and methylene blue as electron acceptor, a decrease absorption at $537 \mathrm{~nm}$ was evident when light was turned on, indicating the formation of reduced methylene blue. Reduction of methylene blue was substantially higher when PSI was encapsulated in PLGA MPs compared with the case of PSI in detergent, consistently with the increased photochemical activity of PSI in PLGA MPs (Figure 3). Even if at this stage it was not possible to assess if methylene blue reduction occurs inside the MPs, by a direct interaction of PSI with the probe, or outside the PLGA MPs through interaction with the PLGA matrix, this result indicates that the encapsulated PSI could be adopted to catalyze light-dependent redox reactions.

\section{DISCUSSION}

In this work the potential use of PSI in extracellular context as a light-dependent redox catalyst was investigated, exploiting the possibility to encapsulate this chlorophyll binding complex in an organic matrix formed by a PLGA polymer. PSI is indeed the photosynthetic complex with the highest quantum efficiency, and it is characterized by fast trapping time of tens of ps: ${ }^{8}$ this complex could thus be considered as an extremely efficient nanomachine that can convert light energy into chemical energy by charge separation. ${ }^{8,32}$ The results herein presented demonstrate that it is possible to encapsulate PSI in PLGA microparticles (Figure 1, Figure 2) maintaining its high photochemical efficiency close to 1 (Supplemental
Table S3). Moreover, PSI encapsulated in PLGA microparticles was characterized by a stronger photochemical activity compared to the detergent solution case (Figure 3), being able to exchange electrons with donors and acceptors added to the MPs mixture. The increased PSI photochemical activity observed in PLGA MPs compared with the detergent case is intriguing because from one side it supports the possible applications of this MPs for the exploitation of PSI photochemical properties, and from the other side, it stimulates a possible hypothesis explaining the effect of PLGA encapsulation on PSI activity. The reason(s) at the base of the increased photochemical activity of PSI in PLGA MPs might be several: the protein-protein interaction formed by PSI in the PLGA MPs might favor electron transfer process and/or PLGA matrix could allow for a more efficient electron transfer to and from the PSI complex. Encapsulation of PSI in PLGA MPs did not alter the light-harvesting properties of PSI but caused a broadening of the absorption spectrum with a stronger emission above $700 \mathrm{~nm}$ compared to PSI in detergent solution. This spectroscopic feature is usually related to a partial aggregation state of chlorophyll binding complexes: ${ }^{50}$ in this condition, the different PSI complexes could thus be more interconnected creating an environment where charge separation at the level of P700 reaction centers is facilitated compared to the PSI monomers found in detergent solutions. A partial aggregation state of PSI could also be more similar to the in vivo condition, where the PSI complexes are present in the thylakoid membranes, which have been reported to be densely crowded by photosynthetic proteins. ${ }^{55}$ Alternatively, since PSI is a membrane complex, formed by protein subunits with several hydrophobic domains, the protein-protein interaction induced in PLGA MPs could stabilize the complex conformation to a more photochemical active state compared with PSI in detergent. PSI in PLGA MPs catalyze a lightdependent electron transport chain from electron donors to electron acceptors: ascorbate was successfully adopted as electron donor, while both methyl-viologen and methylene blue were used as electron acceptors. Considering the porous structure of the PLGA matrix, ${ }^{42}$ it is likely that electron acceptors and donors can diffuse inside the MPs and that the redox reactions occur upon direct contact with encapsulated PSI. However, it cannot be excluded that the PLGA matrix surrounding PSI could mediate the electron exchange between PSI and electron donors and/or acceptors. The PLGA matrix forming the MPs herein investigated is indeed characterized by negative charges, which could be the source of electrons for rereduction of P700 reaction center and/or reduction of the electron acceptors when electrons transported by PSI are available for regenerating the negative charges on the organic layer. Further investigations are required to evaluate the possible role of PLGA on the electron transport induced by light-dependent charge separation at the level of P700. Additionally, the encapsulation of PSI in chitosan MPs, characterized by positive superficial charges, did not increase PSI photochemical activity and stability as in the case of PLGA matrix, suggesting that the presence of negative charge on the MPs surface might be important for activity and stability of the encapsulated PSI. Indeed, encapsulation of PSI complexes in PLGA matrix conferred an additional stability compared with PSI in detergent solution. This enhanced stability for PSI embedded in PLGA MPs is likely ascribable to the amphiphilic nature of the polymer itself ensuring a hydrophobic environment as experienced by PSI in the thylakoid membranes. 
Conversely, chitosan MPs are not able to ensure a hydrophobic environment and PSI complexes even if embedded in the MPs and in a hydrophilic environment, leading to a possible easiest destabilization due to loss of protein conformation. PSI PLGA MPs maintained the same photochemical efficiency after 10 days of strong illumination, and after more than 30 days, a residual $30 \%$ photochemical activity was still detectable. Moreover, encapsulated PSI could be safely lyophilized with a minor reduction of its photochemical properties, observing P700 oxidation even in lyophilized PLGA MPs. The preservation of a functional PSI complex functionality after lyophilization represents an important achievement for the applications of PSI in PLGA MPs and for their storage and transportation.

Furthermore, these microstructures might represent new potential items to produce energy: they could be employed in photovoltaic panels and/or they could be used in the $\mathrm{H}_{2}$ production if coupled with a hydrogenase enzyme, ${ }^{23}$ exploiting electron transport induced by PSI photochemistry to produce $\mathrm{H}_{2}$ from $\mathrm{H}^{+}$. In addition, it is important to note that a disadvantage in using MPs is that efficient coupling of PSI complexes with photovoltaic devices require proper orientation of PSI, ${ }^{12}$ which is difficult to achieve in MPs. Several previous works reported the application of PSI in an extracellular environment coupled with artificial devices, ${ }^{15,16,20-22,24}$ carbon nanotubes, ${ }^{56}$ metal oxide nanowires, ${ }^{57}$ plasmonic nanostructures, ${ }^{34-36}$ conductive polymers, ${ }^{58}$ redox hydrogel, ${ }^{18,59}$ or assembled in metal-organic framework. ${ }^{32}$ The advantage of the technology herein proposed, in addition to the increased photochemical activity and increased stability of PSI, consists of the organic matrix used for PSI encapsulation: PLGA polymers are environmental friendly compounds since they are biocompatible, ${ }^{41,43}$ and their ester linkages are rapidly hydrolyzed once they enter a cell to produce the monomers, lactic acid and glycolic acid, which are then easily metabolized via the Krebs cycle. PLGA-based MPs have been indeed approved by U.S. Food and Drug Administration (FDA) and by the European Medicine Agency (EMA) ${ }^{41}$ as a delivery vehicle for drugs, proteins, and other molecules such as DNA, RNA, and peptides. In this context, it is possible to conceive a potential application of PSI MPs interacting with animal/ human cells or biomimetic system to specifically deliver lightdependent redox reactions.

\section{ASSOCIATED CONTENT}

\section{S Supporting Information}

The Supporting Information is available free of charge on the ACS Publications website at DOI: 10.1021/acssuschemeng.9b00738.

Table S1. HPLC analysis of PSI in detergent and in PLGA MPs. Table S2. Z-Average value, polydispersity index (PDI) and superficial charge of PLGA and chitosan microparticles. Table S3. Fitting analysis of florescence decay integrated kinetics. Figure S1. PSI encapsulation in PLGA MPs. Figure S2. Atomic Force Microscopy (AFM) observation of PLGA empty microparticles and particle size distribution. Figure S3. Power dependency of fluorescence kinetics of PSI in PLGA MPs Figure S4. Isothermal DSC analysis. Figure S5. PSI encapsulation in chitosan MPs. Figure S6. Atomic Force Microscopy (AFM) observation of PSI in chitosan microparticles. Figure S7. Absorption and fluorescence emission spectra of PSI in detergent and in chitosan MPs. Figure S8. Photochemical activity of PSI in detergent vs PLGA or chitosan microparticles. Figure S9. Stability of PSI to light exposure. Figure S10. P700 oxidation of lyophilized microparticles containing Photosystem I. Figure S11. Absorption spectrum of PSI microparticles before and after lyophilization (PDF)

\section{AUTHOR INFORMATION}

\section{Corresponding Authors}

*Telephone: +39 0458027807. E-mail: matteo.ballottari@ univr.it.

*Telephone: +39 0458027984. E-mail: massimiliano. perduca@univr.it.

\section{ORCID}

Matteo Ballottari: 0000-0001-8410-3397

\section{Author Contributions}

M.P. and M.Ba. conceived the work; M.P. supervised the nanoparticles synthesis; M.B. supervised the purification and spectroscopic characterization of PSI; A.C., L.D.S., and M.Bo. performed the nanoparticle synthesis and characterization; A.R. performed the AFM experiments and analyzed the data; F.P., I.B., and C.D. performed the time-resolved fluorescence analysis; M.Ba. and M.P. wrote the paper; all the authors discussed the results and commented on the manuscript.

\section{Funding}

The research was supported by the ERC Starting Grant SOLENALGAE (679814) to M.Ba.

\section{Notes}

The authors declare no competing financial interest.

\section{ACKNOWLEDGMENTS}

Authors would like to thank Prof. Roberto Bassi from the University of Verona for giving the possibility to use JTS-10 BioLogic instrument. The research was supported by the ERC Starting Grant SOLENALGAE (679814) to M.Ba.

\section{ABBREVIATIONS}

PSI, photosystem I; PSII, photosystem II; PLGA, poly lacticco-glycolic acid; MPs, microparticles; AFM, atomic force microscopy; DSC, differential scanning calorimetry; FDA, U.S. Food and Drug Administration; EMA, European Medicine Agency; TPP, tripolyphosphate; PVA, poly(vinyl alcohol); DLS, dynamic light scattering.

\section{REFERENCES}

(1) Ben-Shem, A.; Frolow, F.; Nelson, N. Crystal structure of plant photosystem I. Nature 2003, 426 (6967), 630-635.

(2) Wei, X.; Su, X.; Cao, P.; Liu, X.; Chang, W.; Li, M.; Zhang, X.; Liu, Z. Structure of spinach photosystem II-LHCII supercomplex at 3.2 Å resolution. Nature 2016, 534 (7605), 69-74.

(3) Caffarri, S.; Tibiletti, T.; Jennings, R. C.; Santabarbara, S. A comparison between plant photosystem I and photosystem II architecture and functioning. Curr. Protein Pept. Sci. 2014, 15i (4), 296-331.

(4) Umena, Y.; Kawakami, K.; Shen, J. R.; Kamiya, N. Crystal structure of oxygen-evolving photosystem II at a resolution of $1.9 \AA$. Nature 2011, 473 (7345), 55-60.

(5) Mazor, Y.; Borovikova, A.; Nelson, N. The structure of plant photosystem I super-complex at 2.8 Å resolution. eLife 2015, 4, 1-18.

(6) Qin, X.; Suga, M.; Kuang, T.; Shen, J. R. Photosynthesis. Structural basis for energy transfer pathways in the plant PSI-LHCI supercomplex. Science 2015, 348 (6238), 989-995. 
(7) Tikhonov, A. N. The cytochrome b6f complex at the crossroad of photosynthetic electron transport pathways. Plant Physiol. Biochem. 2014, 81, 163-183.

(8) Croce, R.; van Amerongen, H. Light-harvesting in photosystem I. Photosynth. Res. 2013, 116 (2-3), 153-166.

(9) van Amerongen, H.; Croce, R. Light harvesting in photosystem II. Photosynth. Res. 2013, 116 (2-3), 251-263.

(10) Werst, M.; Jia, Y.; Mets, L.; Fleming, G. R. Energy transfer and trapping in the photosystem I core antenna. A temperature study. Biophys. J. 1992, 61 (4), 868-878.

(11) Wientjes, E.; van Stokkum, I. H.; van Amerongen, H.; Croce, R. Excitation-energy transfer dynamics of higher plant photosystem I light-harvesting complexes. Biophys. J. 2011, 100 (5), 1372-1380.

(12) Frolov, L.; Rosenwaks, Y.; Carmeli, C.; Carmeli, I. Fabrication of a photoelectronic device by direct chemical binding of the photosynthetic reaction center protein to metal surfaces. Adv. Mater. 2005, 17 (20), 2434-2437.

(13) Kaniber, S. M.; Simmel, F. C.; Holleitner, A. W.; Carmeli, I. The optoelectronic properties of a photosystem I-carbon nanotube hybrid system. Nanotechnology 2009, 20 (34), 345701.

(14) Carmeli, I.; Mangold, M.; Frolov, L.; Zebli, B.; Carmeli, C.; Richter, S.; Holleitner, A. A photosynthetic reaction center covalently bound to carbon nanotubes. Adv. Mater. 2007, 19 (22), 3901-3905.

(15) Carmeli, I.; Frolov, L.; Carmeli, C.; Richter, S. Photovoltaic activity of photosystem I-based self-assembled monolayer. J. Am. Chem. Soc. 2007, 129 (41), 12352-12353.

(16) Gerster, D.; Reichert, J.; Bi, H.; Barth, J.; Kaniber, S.; Holleitner, A.; Visoly-Fisher, I.; Sergani, S.; Carmeli, I. Photocurrent of a single photosynthetic protein. Nat. Nanotechnol. 2012, 7 (10), 673-676.

(17) Zhao, F.; Hardt, S.; Hartmann, V.; Zhang, H.; Nowaczyk, M.; Rogner, M.; Plumere, N.; Schuhmann, W.; Conzuelo, F. Lightinduced formation of partially reduced oxygen species limits the lifetime of photosystem 1-based biocathodes. Nat. Commun. 2018, 9, 1973.

(18) Zhao, F.; Conzuelo, F.; Hartmann, V.; Li, H.; Nowaczyk, M.; Plumere, N.; Rogner, M.; Schuhmann, W. Light Induced H-2 Evolution from a Biophotocathode Based on Photosystem 1-Pt Nanoparticles Complexes Integrated in Solvated Redox Polymers Films. J. Phys. Chem. B 2015, 119 (43), 13726-13731.

(19) Kothe, T.; Poller, S.; Zhao, F.; Fortgang, P.; Rogner, M.; Schuhmann, W.; Plumere, N. Engineered Electron-Transfer Chain in Photosystem 1 Based Photocathodes Outperforms Electron-Transfer Rates in Natural Photosynthesis. Chem. - Eur. J. 2014, 20 (35), 11029-11034.

(20) Zeynali, A.; Ghiasi, T.; Riazi, G.; Ajeian, R. Organic solar cell based on photosystem I pigment-protein complex, fabrication and optimization. Org. Electron. 2017, 51, 341-348.

(21) Gunther, D.; LeBlanc, G.; Prasai, D.; Zhang, J. R.; Cliffel, D. E.; Bolotin, K. I.; Jennings, G. K. Photosystem I on graphene as a highly transparent, photoactive electrode. Langmuir 2013, 29 (13), 41774180.

(22) Das, R.; Kiley, P.; Segal, M.; Norville, J.; Yu, A.; Wang, L.; Trammell, S.; Reddick, L.; Kumar, R.; Stellacci, F.; Lebedev, N.; Schnur, J.; Bruce, B.; Zhang, S.; Baldo, M. Integration of photosynthetic protein molecular complexes in solid-state electronic devices. Nano Lett. 2004, 4 (6), 1079-1083.

(23) Krassen, H.; Schwarze, A.; Friedrich, B.; Ataka, K.; Lenz, O.; Heberle, J. Photosynthetic hydrogen production by a hybrid complex of photosystem I and [NiFe]-hydrogenase. ACS Nano 2009, 3 (12), 4055-4061.

(24) Kuritz, T.; Lee, I.; Owens, E.; Humayun, M.; Greenbaum, E. Molecular photovoltaics and the photoactivation of mammalian cells. Ieee Transactions on Nanobioscience 2005, 4 (2), 196-200.

(25) Di Maria, F.; Lodola, F.; Zucchetti, E.; Benfenati, F.; Lanzani, G. The evolution of artificial light actuators in living systems: from planar to nanostructured interfaces. Chem. Soc. Rev. 2018, 47 (13), $4757-4780$.
(26) Saboe, P.; Conte, E.; Farell, M.; Bazan, G.; Kumar, M. Biomimetic and bioinspired approaches for wiring enzymes to electrode interfaces. Energy Environ. Sci. 2017, 10 (1), 14-42.

(27) Saboe, P.; Conte, E.; Chan, S.; Feroz, H.; Ferlez, B.; Farell, M.; Poyton, M.; Sines, I.; Yan, H.; Bazan, G.; Golbeck, J.; Kumar, M. Biomimetic wiring and stabilization of photosynthetic membrane proteins with block copolymer interfaces. J. Mater. Chem. A 2016, 4 (40), 15457-15463.

(28) Saboe, P.; Lubner, C.; McCool, N.; Vargas-Barbosa, N.; Yan, H.; Chan, S.; Ferlez, B.; Bazan, G.; Golbeck, J.; Kumar, M. TwoDimensional Protein Crystals for Solar Energy Conversion. Adv. Mater. 2014, 26 (41), 7064-7069.

(29) Niroomand, H.; Pamu, R.; Mukherjee, D.; Khomami, B. Microenvironment alterations enhance photocurrents from photosystem I confined in supported lipid bilayers. J. Mater. Chem. A 2018, 6 (26), 12281-12290.

(30) Niroomand, H.; Pamu, R.; Mukherjee, D.; Khomami, B. Tuning the photocurrent generations from photosystem I assembled in tailored biotic-abiotic interfaces. MRS Commun. 2018, 8 (3), 823829.

(31) Niroomand, H.; Mukherjee, D.; Khomami, B. Tuning the photoexcitation response of cyanobacterial Photosystem I via reconstitution into Proteoliposomes. Sci. Rep. 2017, 7, 2492.

(32) Bennett, T. H.; Vaughn, M. D.; Davari, S. A.; Park, K.; Mukherjee, D.; Khomami, B. Jolly green MOF: confinement and photoactivation of photosystem I in a metal-organic framework. Nanoscales Advances 2019, 1, 94-104.

(33) Carmeli, I.; Lieberman, I.; Kraversky, L.; Fan, Z.; Govorov, A. O.; Markovich, G.; Richter, S. Broad band enhancement of light absorption in photosystem I by metal nanoparticle antennas. Nano Lett. 2010, 10 (6), 2069-2074.

(34) Pamu, R.; Sandireddy, V.; Kalyanaraman, R.; Khomami, B.; Mukherjee, D. Plasmon-Enhanced Photocurrent from Photosystem I Assembled on Ag Nanopyramids. J. Phys. Chem. Lett. 2018, 9 (5), 970-977.

(35) Czechowski, N.; Lokstein, H.; Kowalska, D.; Ashraf, K.; Cogdell, R.; Mackowski, S. Large plasmonic fluorescence enhancement of cyanobacterial photosystem I coupled to silver island films. Appl. Phys. Lett. 2014, 105 (4), 043701.

(36) Brecht, M.; Hussels, M.; Nieder, J.; Fang, H.; Elsasser, C. Plasmonic interactions of photosystem I with Fischer patterns made of Gold and Silver. Chem. Phys. 2012, 406, 15-20.

(37) Kim, I.; Bender, S. L.; Hranisavljevic, J.; Utschig, L. M.; Huang, L.; Wiederrecht, G. P.; Tiede, D. M. Metal nanoparticle plasmonenhanced light-harvesting in a photosystem I thin film. Nano Lett. 2011, 11 (8), 3091-3098

(38) Lü, J. M.; Wang, X.; Marin-Muller, C.; Wang, H.; Lin, P. H.; Yao, Q.; Chen, C. Current advances in research and clinical applications of PLGA-based nanotechnology. Expert Rev. Mol. Diagn. 2009, i9 (4), 325-341.

(39) Ali, M. E.; Lamprecht, A. Polyethylene glycol as an alternative polymer solvent for nanoparticle preparation. Int. J. Pharm. 2013, 456 (1), 135-142.

(40) Danhier, F.; Ansorena, E.; Silva, J. M.; Coco, R.; Le Breton, A.; Préat, V. PLGA-based nanoparticles: an overview of biomedical applications. J. Controlled Release 2012, 161 (2), 505-522.

(41) Makadia, H. K.; Siegel, S. J. Poly Lactic-co-Glycolic Acid (PLGA) as Biodegradable Controlled Drug Delivery Carrier. Polymers (Basel, Switz.) 2011, i3 (3), 1377-1397.

(42) Klose, D.; Siepmann, F.; Elkharraz, K.; Krenzlin, S.; Siepmann, J. How porosity and size affect the drug release mechanisms from PLGA-based microparticles. Int. J. Pharm. 2006, 314 (2), 198-206.

(43) Kumari, A.; Yadav, S. K.; Yadav, S. C. Biodegradable polymeric nanoparticles based drug delivery systems. Colloids Surf., B 2010, 75 (1), $1-18$.

(44) Wang, L. Y.; Gu, Y. H.; Zhou, Q. Z.; Ma, G. H.; Wan, Y. H.; Su, Z. G. Preparation and characterization of uniform-sized chitosan microspheres containing insulin by membrane emulsification and a 
two-step solidification process. Colloids Surf., B 2006, 50 (2), 126135.

(45) Barichello, J. M.; Morishita, M.; Takayama, K.; Nagai, T. Encapsulation of hydrophilic and lipophilic drugs in PLGA nanoparticles by the nanoprecipitation method. Drug Dev. Ind. Pharm. 1999, 25 (4), 471-476.

(46) Ballottari, M.; Govoni, C.; Caffarri, S.; Morosinotto, T. Stoichiometry of LHCI antenna polypeptides and characterization of gap and linker pigments in higher plants Photosystem I. Eur. J. Biochem. 2004, 271 (23-24), 4659-4665.

(47) Ballottari, M.; Alcocer, M. J.; D’Andrea, C.; Viola, D.; Ahn, T. K.; Petrozza, A.; Polli, D.; Fleming, G. R.; Cerullo, G.; Bassi, R. Regulation of photosystem I light harvesting by zeaxanthin. Proc. Natl. Acad. Sci. U. S. A. 2014, 111 (23), E2431-E2438.

(48) Galka, P.; Santabarbara, S.; Khuong, T. T.; Degand, H.; Morsomme, P.; Jennings, R. C.; Boekema, E. J.; Caffarri, S. Functional analyses of the plant photosystem I-light-harvesting complex II supercomplex reveal that light-harvesting complex II loosely bound to photosystem II is a very efficient antenna for photosystem I in state II. Plant Cell 2012, 24 (7), 2963-2978.

(49) Lagarde, D.; Beuf, L.; Vermaas, W. Increased production of zeaxanthin and other pigments by application of genetic engineering techniques to Synechocystis sp. strain PCC 6803. Appl. Environ. Microbiol. 2000, 66 (1), 64-72.

(50) Horton, P.; Ruban, A. V.; Rees, D.; Pascal, A. A.; Noctor, G.; Young, A. J. Control of the light-harvesting function of chloroplast membranes by aggregation of the LHCII chlorophyll-protein complex. FEBS Lett. 1991, 292 (1-2), 1-4.

(51) Novoderezhkin, V. I.; Croce, R.; Wahadoszamen, M.; Polukhina, I.; Romero, E.; van Grondelle, R. Mixing of exciton and charge-transfer states in light-harvesting complex Lhca4. Phys. Chem. Chem. Phys. 2016, 18 (28), 19368-19377.

(52) Joliot, P.; Johnson, G. N. Regulation of cyclic and linear electron flow in higher plants. Proc. Natl. Acad. Sci. U. S. A. 2011, 108 (32), 13317-13322.

(53) Bonente, G.; Pippa, S.; Castellano, S.; Bassi, R.; Ballottari, M. Acclimation of Chlamydomonas reinhardtii to different growth irradiances. J. Biol. Chem. 2012, 287 (8), 5833-5847.

(54) Edison, T.; Sethuraman, M. Instant green synthesis of silver nanoparticles using Terminalia chebula fruit extract and evaluation of their catalytic activity on reduction of methylene blue. Process Biochem. 2012, 47 (9), 1351-1357.

(55) Goral, T. K.; Johnson, M. P.; Brain, A. P.; Kirchhoff, H.; Ruban, A. V.; Mullineaux, C. W. Visualizing the mobility and distribution of chlorophyll proteins in higher plant thylakoid membranes: effects of photoinhibition and protein phosphorylation. Plant J. 2010, 62 (6), 948-959.

(56) Kaniber, S. M.; Brandstetter, M.; Simmel, F. C.; Carmeli, I.; Holleitner, A. W. On-chip functionalization of carbon nanotubes with photosystem I. J. Am. Chem. Soc. 2010, 132 (9), 2872-2873.

(57) Mershin, A.; Matsumoto, K.; Kaiser, L.; Yu, D.; Vaughn, M.; Nazeeruddin, M. K.; Bruce, B. D.; Graetzel, M.; Zhang, S. Selfassembled photosystem-I biophotovoltaics on nanostructured $\mathrm{TiO}(2)$ and ZnO. Sci. Rep. 2012, 2, 234.

(58) Robinson, M.; Simons, C.; Cliffel, D.; Jennings, G. Photocatalytic photosystem I/PEDOT composite films prepared by vaporphase polymerization. Nanoscale 2017, i9 (18), 6158-6166.

(59) Badura, A.; Guschin, D.; Kothe, T.; Kopczak, M.; Schuhmann, W.; Rogner, M. Photocurrent generation by photosystem 1 integrated in crosslinked redox hydrogels. Energy Environ. Sci. 2011, i4 (7), $2435-2440$. 